\title{
EL PERIODISTA COMO AGENTE SOCIAL: PROPUESTA DIDÁCTICA PARA LA INTERVENCIÓN COMUNICATIVA EN COMUNIDADES CERCANAS
}

\author{
The journalist as a social agent: A didactic proposal \\ for their communicative intervention in neighbor \\ communities
}

\section{Vanesa Saiz-Echezarreta y Belén Galletero-Campos}
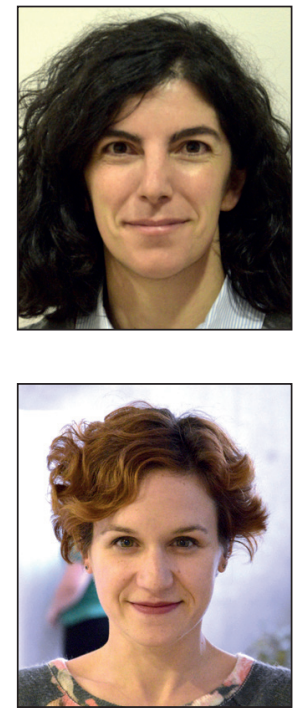

Vanesa Saiz-Echezarreta es doctora en periodismo por la Universidad Complutense de Madrid, y actualmente profesora y vicedecana en la Facultad de Periodismo de la Universidad de Castilla La Mancha. Pertenece al grupo de investigación Semiótica, Comunicación y Cultura (UCM) y es especialista en semiótica, estudios de género y análisis socio-cultural. Desarrolla su investigación en el Proyecto Issues, sobre la mediatización de los asuntos públicos en la esfera mediatizada, ocupándose de la controversia sobre prostitución e imaginarios socio-sexuales.

http://orcid.org/0000-0003-1700-0296

vanesa.saiz@uclm.es

Belén Galletero-Campos es licenciada en Periodismo por la Universidad de Navarra, experta en guion de ficción para TV y cine por la Universidad Pontificia de Salamanca y master en Comunicación política y empresarial por la Universidad Camilo José Cela. Ha trabajado como periodista en medios de comunicación y como directora de comunicación en varias empresas. Es doctoranda en la Facultad de Periodismo de la Universidad de Castilla-La Mancha donde imparte varias asignaturas y forma parte del grupo de investigación MediaCom.

http://orcid.org/0000-0002-9549-9507

belen.galletero@uclm.es

Universidad de Castilla-La Mancha, Facultad de Periodismo Aulario Polivalente Campus Universitario, s/n. 16071 Cuenca, España

\section{Resumen}

En los últimos años se debate acerca de los perfiles profesionales emergentes y las competencias requeridas en el periodismo. No obstante, en ocasiones se olvida que su función básica de mediación sigue vigente, adaptada, eso sí, a los acelerados avances tecnológicos y a los cambios socioculturales aparejados. Exploramos en qué sentido la vocación de servicio y el papel articulador del periodismo para determinadas comunidades exigen competencias profesionales adaptadas al contexto tecnológico. Deben situar la ética y la responsabilidad como valores centrales del ejercicio periodístico, sin dejarse llevar por la fascinación de las transformaciones técnicas. Proponemos algunas estrategias didácticas para incorporarlas a los programas de estudios universitarios.

\section{Palabras clave}

Periodismo; Periodistas; Mediación; Comunidades de referencia; Periodismo social; Propuestas didácticas; Nuevas tecnologías; Medios sociales; Aprendizaje situado.

\section{Abstract}

In recent years there has been a debate about the emerging professional profiles and skills required in journalism. However, it sometimes goes unnoticed that the basic function of mediation between the facts and the readers in journalism is still valid, although it must be adapted to rapid technological advances and sociocultural changes. We explore how the vocation of service and the coordinating role of journalism for certain communities requires professional skills adapted to the technological context, but not blinded by the fascination of technical transformations. Ethics and responsibility must be the core values in the exercise of journalism. We propose some teaching methods to incorporate into the university curriculum.

\section{Keywords}

Journalism; Journalists; Mediation; Reference communities; Social journalism; Didactic proposals; New technologies; Social media; Situated learning. 
Saiz-Echezarreta, Vanesa; Galletero-Campos, Belén (2017). “El periodista como agente social: propuesta didáctica para la intervención comunicativa en comunidades cercanas”. El profesional de la información, v. 26, n. 1, pp. 125-131.

https://doi.org/10.3145/epi.2017.ene.13

\section{Introducción}

El contexto de convergencia cultural ha abierto amplias posibilidades de interacción y participación ciudadana que están siendo analizadas y experimentadas desde la perspectiva del periodismo y la comunicación. Algunos autores se refieren al hecho de que los periodistas han perdido el privilegio de la mediación (Mancini, 2012) y deben repensar su función social. Al mismo tiempo, siguiendo una perspectiva de determinismo tecnológico (Aibar, 1996, 2001) se dibuja un panorama en el que los ciudadanos se ven obligados a autoorganizarse (Castells, 2009, p. 87).

\section{El periodismo está obligado a redefinir sus modos de actuar, pero sin perder de vista los aspectos que siguen legitiman- do su función social}

No obstante, quizá sería preferible no dar tantas cosas por supuestas: muchas de las dinámicas que suceden en las redes sociales siguen siendo mediocéntricas. El periodismo mantiene

"una función principal de informar, de la que derivan directamente otras dos: la acción intrínseca de comunicar la interpretación que construye de la realidad, y la labor de integración y mediación con la sociedad al difundir informaciones, interpretaciones y opiniones" (SánchezGarcía; Campos-Domínguez; Berrocal-Gonzalo, 2015, p. 196).

La función social del periodismo se ha transformado, pero ¿hasta qué punto? Si aplicamos una mirada centrada en la remediación (Bolter; Grusin, 2000) y la agencia compartida (Latour, 2008), la convergencia tecnológica incide en nuestra forma de contar la realidad, de aproximar versiones del mundo, espacios, valores comunes y compartidos. Sin embargo no estamos ante algo nuevo, estamos aprendiendo a remediar, a traducir aquello que tenemos encomendado como tarea de la profesión periodística a los nuevos entornos y herramientas.

Kapucinski (2003, p. 16) recuerda la centralidad del cuidado en el periodismo, dado que

"tenemos que entender que se trata de una obra colectiva en la que participan las personas de quienes obtuvimos las informaciones y opiniones con las que realizamos nuestro trabajo. Por supuesto que un periodista debe tener cualidades propias, pero su tarea va a depender de los otros: aquel que no sabe compartir, difícilmente puede dedicarse a esta profesión".

El periodismo está obligado a redefinir su modo de actuar, pero sin perder de vista los aspectos que siguen legitimando su función social, entre ellos, la perspectiva ética y el objetivo de defender los valores de justicia y profundización democrática. En este quehacer, la atención hacia los públicos que tienen menos espacios de participación en el espacio público, cuando no están siendo silenciados y/o estigmatizados, cobra un valor central. Más aún cuando los receptores empiezan a superar el concepto de audiencia para concebirse como públicos y comunidades.

Este artículo explora algunos métodos didácticos que podrían utilizarse en el marco de procesos de enseñanzaaprendizaje que se resisten a la fascinación tecnológica, afrontan la propia vulnerabilidad y los límites a partir del autoanálisis y apuestan por el cuidado como perspectiva ética del trabajo con los colectivos en situación de desventaja.

\section{El debate de las competencias}

Fenómenos como las redes sociales (Facebook, 2004; Twitter, 2006; YouTube, 2005) (López-García, 2015) son coetáneos al Libro blanco. Títulos de grado en comunicación de Aneca (2005). Con los nuevos roles asociados al periodista cabría pensar en una inminente revisión del perfil formativo de este profesional acorde con las nuevas realidades. En los últimos años, muy en especial a partir de la adaptación de los grados al Espacio Europeo de Educación Superior, se ha producido un debate (Tuñez-López; Martínez-Solana; Abejón-Mendoza, 2010; Scolari et al., 2008; Gertrudis-Casado; Gértrudix; Álvarez-García, 2016; Sánchez-García; CamposDomínguez; Berrocal, 2015) sobre el modelo de competencias que deben adquirir los periodistas.

Para cumplir con el reto de adecuarse a un ecosistema mediático que experimenta transformaciones constantes y veloces, las investigaciones identifican dos aspectos clave:

- la especialización tecnológica (Tuñez-López; Martínez-Solana; Abejón-Mendoza, 2010) y la innovación (Salaverría, 2015);

- la faceta empresarial del periodista como promotor de su propio medio, o periodismo emprendedor (ManfrediSánchez, 2015).

Estos modelos tienden a explorar nuevos formatos multimedia vinculados a la búsqueda de nuevas oportunidades de negocio pero no es frecuente que la innovación se asocie al papel del periodista en el proceso.

Este encuadre es coherente con la orientación práctica de los estudios de periodismo, focalizados en la aplicabilidad directa, frente a lo que sucede en otros estudios de ciencias sociales en los que la reflexión teórica y deontológica tiene un mayor peso. Prueba de ello es que si observamos la distribución de competencias en el Libro blanco, aquellas que indican la importancia de la función social del periodismo en la profundización democrática quedan en un apartado específico, señalando así su carácter excepcional. Su carác- 
ter transversal, a priori positivo, puede propiciar que finalmente no se incorporen a las materias regladas, quedando al arbitrio de cada docente.

En este marco las competencias que facilitan a los periodistas atender la vulnerabilidad, las situaciones de injusticias y la violación de derechos humanos podrían perder relevancia en favor de otras que apelan a la eficacia y la eficiencia, que se perciben como menos conflictivas, requieren menor innovación pedagógica y no están dotadas de una dimensión política, en su sentido más amplio. Veamos por ejemplo los resultados del estudio sobre competencias de Casero-Ripollés, Ortells-Badenes y Doménech-Fabregat (2013). Para alumnos y profesionales las competencias esenciales tienen que ver con conocer los acontecimientos de la actualidad periodística y aplicar criterios de noticiabilidad, realizar prácticas durante el período formativo y gestionar fuentes de información. Sin embargo, el conocimiento de los fundamentos teóricos del periodismo o, lo que es más relevante por cuanto puede influir en el entorno, sus implicaciones sociales y políticas, quedan en el espacio de lo recomendable pero no imprescindible.

A un community manager se le presuponen actitudes como capacidad de escucha, transparencia y reflexión, cualidades no necesariamente vinculadas a publicidad y relaciones públicas

Nuestra propuesta es cuestionar que estas capacidades prácticas y tecnológicas deban ser las únicas o al menos las hegemónicas. Partiendo de la triple dimensión de las competencias que propone Riesco-González (2008, p. 91), "el conocimiento conceptual, la técnica instrumental y el compromiso personal y la actitud", planteamos que el refuerzo del ámbito competencial basado en actitudes ayudará a devolver al periodismo su función social y mediadora. Con este objetivo creemos que la educación profesional tiene que ofrecer herramientas al alumnado para que pueda trabajar sobre el terreno implicándose en su contexto más cercano abriendo el pensamiento a la pregunta: ¿Qué puedo hacer yo como periodista para mejorar mi comunidad? (Jarvis, 2015).

Esto obliga a desviar el foco desde una mirada más empresarial o marketiniana vinculada al periodismo emprendedor, hacia un modelo colaborativo con vocación de servicio, en marcos más amplios que pueden incluir la economía social, el tercer sector o el sector público, complementando a las economías de mercado. Es decir, profundizar en la confluencia de los enfoques que se han propuesto desde diversas perspectivas complementarias como la educomunicación, el periodismo de paz, la comunicación para el cambio social, etc. (Nos-Aldás, 2011; Nos-Aldás; Smith, 2016; OrozcoGómez; Navarro-Martínez; García-Matilla, 2012), pero no de manera colateral a través de una asignatura, sino como parte de proyectos cotidianos para cualquier periodista.

Esta visión cobra aún más importancia cuando sabemos que la brecha digital ha traído consigo nuevas formas de vulne- rabilidad que deben ser abordadas. Nos encontramos, como plantea Arriazu-Muñoz (2015), en una sociedad del conocimiento cada vez más selectiva en el uso y manejo de la tecnología. En este nuevo contexto comunicativo, en el que por cada individuo hay un relato, "hemos pasado de una idea objetiva de la realidad al territorio de la subjetividad" (Bassets, 2013, p. 85), en la que el individuo escoge cuál es su visión de la misma, una espiral egocentrista que puede dejar fuera aquello que no queremos ver. Este fenómeno está agudizado por las posibilidades tecnológicas de la personalización extrema y la segmentación de los mercados.

La labor de los comunicadores -entre ellos, los periodistasen la tarea de evitar el aislamiento de las comunidades y facilitar la comprensión mutua es fundamental. Necesitamos de la información para construir vínculo social y para expandir nuestras capacidades en el espacio público, tanto las de deliberación democrática, como las de actuación colectiva en el ejercicio del poder. Asimismo la labor periodística es imprescindible para movilizar un trabajo de imaginación colectiva acerca del mundo que está por construir; de ahí que los periodistas puedan y deban asumir su papel en promover una convivencia más igualitaria y participativa (GámezFuentes; Nos-Aldás, 2012).

La existencia de una comunidad de afectados legitimada en el espacio público depende de su lucha por hacerse oír (Lafuente; Lara, 2013, p. 174). El esfuerzo de una comunidad por hacerse visible comienza con identificar la naturaleza del problema, plantearlo en una narrativa comprensible y conseguir que tenga eco (Lafuente; Corsin, 2015). Esta fórmula ya se viene aplicando en ámbitos del periodismo como la comunicación corporativa, pero al desplazar la perspectiva empresarial hacia la social, el periodista debe adquirir un compromiso de manera que se genere un vínculo entre el profesional y los colectivos implicados en un modo capaz de superar los roles clásicos de fuentes y audiencias.

\section{Herramientas y competencias para cumplir con la tarea mediadora}

La preocupación por la comunidad está en boga en los enfoques actuales sobre la profesión periodística. Asistimos al auge de nuevos perfiles (Martínez-Rodrigo; Sánchez-Martín, 2012, pp. 129-130):

- planificador de medios sociales

- responsable de reputación digital

- SEO

- gestor de contenidos...

Todos ellos vinculados al mundo empresarial en el que el fin último es la captación de clientes, la fidelización de la comunidad de usuarios y la creación digital de imagen de marca, avanzando un paso más en la comunicación corporativa tradicional. Pero ¿̇qué sucede cuando el objetivo no es vender o lanzar un producto sino atender necesidades de un grupo de ciudadanos?

Según la propia etimología del término, un community manager es el gestor de comunidades online, tarea para la que se requiere, "capacidad de escucha, transparencia, reflexión, actitud crítica, liderazgo y vocación" (Castelló- 
Martínez, 2010, p. 87), cualidades no necesariamente vinculadas al ámbito de la publicidad y las relaciones públicas. Recuperamos el ejemplo que plantean Espíritusanto y Gonzalo-Rodríguez (2011, p. 70) acerca del moderador en el proyecto de periodismo colaborativo SoyPeriodista.com de Colombia:

"No es un community manager en el sentido comercial del término, pero sus funciones sí se asemejan en algo a algunas de sus tareas, en cuanto a construcción de comunidad, promoción del diálogo y solución online de posibles conflictos".

La sociedad en red ha confirmado que la necesidad de comunicación no es menor sino incluso mayor, aumentando la sociabilidad de las personas (Castells, 2013, p. 13). En este contexto el periodista tiene un papel relevante y cuenta con más herramientas que nunca. Formatos colaborativos como los wikis podrían ser la plataforma para reconstruir, por ejemplo, la intrahistoria de los pueblos más pequeños de España que han quedado desatendidos por los medios convencionales. Aplicaciones como Storify pueden ser útiles para recoger debates y evaluar sus resultados de cara a plantear acciones dirigidas a un fin. La web 2.0 es una oportunidad para ampliar las funciones del periodismo que ha perdido su función social clásica y debe compartirla con otros actores.

¿Qué método puede ser el más adecuado para atender la vulnerabilidad no en un sentido de atención victimizadora, sino desde la participación responsable? El espacio de mediación con comunidades puede dirigirse a compartir la creación de estrategias de comunicación social sobre la base de la tecnología y la red 2.0, promoviendo la deliberación y el diálogo entre perspectivas y puntos de vista en conflicto. Y hacerlo, no desde un punto de vista supuestamente neutral y distanciado, sino planteando que el disenso sucede -a menudo- en un territorio de valores comunes y un horizonte de expectativas compartidas, que enmarca la controversia y la discusión en torno a necesidades, deseos, propuestas, intereses, etc.

¿Qué sucede cuando el objetivo no es vender o lanzar un producto sino atender necesidades de un grupo de ciudadanos?

Al referirnos a esta mirada ética basada en la sensibilidad moral, el respeto a los otros, no apelamos sólo a los valores. Consideramos que puede traducirse en competencias profesionales concretas, puestas en práctica mediante métodos didácticos adecuados y para ello hemos explorado los espacios comunes en los que el periodismo se entrecruza con el trabajo social. Esta propuesta es de ida y vuelta ya que, al mismo tiempo, los trabajadores sociales no pueden obviar la necesidad de aprender y establecer estrategias de comunicación eficaces en la intervención con las comunidades y colectivos (Aguilar-Idáñez, 2016).

El Libro blanco. Título de grado en trabajo social (Aneca, 2004, pp. 189-192) señala varias competencias que podrían ser aplicables a los estudios periodísticos y desarrolladas mediante un enfoque interdisciplinar a través de proyectos pedagógicos. Destacamos las siguientes:

- apoyar a las personas para que sean capaces de manifestar las necesidades, puntos de vista y circunstancias;

- valorar las necesidades y opciones posibles para orientar una estrategia de intervención;

- utilizar la mediación como estrategia de intervención destinada a la resolución alternativa de conflictos;

- apoyar el desarrollo de redes para hacer frente a las necesidades, y trabajar a favor de los resultados planificados examinando con las personas las redes de apoyo a las que puedan acceder;

- defender a las personas, familias, grupos, organizaciones y comunidades y actuar en su nombre si la situación lo requiere;

- gestionar, presentar y compartir historias e informes sociales manteniéndolos completos, fieles, accesibles y actualizados como garantía en la toma de decisiones y valoraciones profesionales.

Las labores de comunicación, mediación, portavocía y creación de redes están dotadas de una dimensión comunicativa en la que el profesional de la comunicación puede intervenir, no de modo autónomo, sino como parte de equipos interdisciplinares.

La información comprendida como servicio público favorece la función del periodista como mediador, capaz de atender a las necesidades comunicativas de las comunidades hacia el interior y hacia el exterior

\section{Método de trabajo con el alumnado de periodismo}

Este enfoque ya se está aplicando en modelos como el Master en periodismo social de la City University of New York que trabaja con comunidades hiperlocales promoviendo acciones orientadas a mejorar sus relaciones y visibilizar sus problemas en el espacio público. También está relacionado con la propuesta de la profesora Nos-Aldás (2011) de la Universitat Jaume I de Castellón, en su asignatura Discursos audiovisuales y cultura de paz. Sin embargo, se trata de prácticas aún minoritarias que ahondan en un terreno por explorar en nuestro país.

En el caso de la Facultad de Periodismo de la Universidad de Castilla La Mancha, desde hace tres cursos académicos se lleva a cabo en la asignatura Cultura digital, del Grado de Periodismo, una experiencia docente que trata de poner en práctica una primera aproximación a estos planteamientos. Es una asignatura optativa del segundo cuatrimestre del 4 응 curso por lo que los alumnos tendrían que estar ya en disposición de utilizar de modo autónomo las competencias adquiridas. El planteamiento de la asignatura contempla una revisión teórica de materiales que favorecen la reflexión sobre el contexto de cultura de convergencia y los dispositivos 
tecnológicos digitales, desde la perspectiva de la sociología de la tecnología. Se propone a la clase la metodología de aprendizaje por proyectos que persiga dar respuesta a un reto, indagando en sus comunidades cercanas de referencia, para buscar junto a ellas una posible propuesta en relación al reto. Se asemeja así al estudio de caso, basado en:

"la descripción de situaciones y problemas de la vida profesional en torno a los cuales el alumno debe posicionarse (orientación fundamental a la decisión), y que exija un alto nivel de participación e implicación personal de los alumnos" (Fuente-Cobo; Mera-Fernández, 2013, p. 180).

Para cumplir con los objetivos hay dos aspectos previos importantes:

- generar un espacio de trabajo estable, basado en un ambiente de confianza y diálogo entre los participantes, cuyo número se sitúa en torno a las 30 personas;

- desplazar la evaluación del resultado al proceso.

Se plantea al grupo que se trabajará desde la incertidumbre puesto que se desconocen los retos que asumirá la clase y los productos resultantes, contemplando también la posibilidad de que una idea fracase y se bloquee en la fase de indagación.

\section{En un caso real, los estudiantes actuaron como mediadores y animaron a los co- lectivos a hacer una petición conjunta al ayuntamiento usando los medios comu- nicativos a su disposición}

El proceso de enseñanza-aprendizaje persigue articular la actividad intelectual y la familiarización de conceptos teóricos con un trabajo de autoanálisis y reflexión afectiva, para lograr un aprendizaje significativo. Este ejercicio de autoconocimiento se realiza a partir de la autoobservación, el planteamiento de preguntas e intercambio de relatos, así como de la búsqueda de expresión artística de lo aprendido y descubierto, a través de actividades de teatro foro o realización de murales. Consideramos que es un primer paso necesario ya que difícilmente se podrá ayudar a una comunidad o colectivo si el profesional no es capaz de autoexaminarse, ponerse en contacto con sus actitudes, sobre todo los prejuicios, valores y zonas grises que le generan incomodidad e incertidumbre.

La reflexión guiada está orientada a sacar al grupo de la zona de confort, evitando que el discurso derive hacia lugares comunes. Aunque resulte incómodo, es importante confrontar con prejuicios, con los valores implícitos de todo signo (dimensiones identitarias de clase, etnia, edad, orientación sexual, etc.) y, por supuesto, con estilos afectivos y formas de comportamiento.

Volviendo al método de trabajo por proyectos, se advierte al grupo que debe centrarse en la definición del reto a través de una pregunta. A modo de ejemplo, algunas de las propuestas han sido:

- ¿se podrían recuperar espacios abandonados en la ciudad para la promoción de actividades culturales?;
- ¿se puede reducir la violencia en el deporte base?;

- ¿cómo apoyar a los mayores en la reducción de la brecha digital?;

- ¿cómo conseguir que la perspectiva de la diversidad funcional sea más conocida en la ciudad frente al marco de la minusvalía?;

- ¿es posible visibilizar y revalorizar en el espacio público trabajos estigmatizados como los de la limpieza?

Una de las mayores dificultades es que los grupos no son capaces de focalizar sobre el reto, sino que se concentran de inmediato en el producto que desarrollarán (un reportaje, una guía, una campaña, etc.). La actitud adoptada por el alumnado corresponde a la de profesionales que sensibilizarán a otros, sustituyendo la perspectiva múltiple de las necesidades de las comunidades de referencia por su propia visión de lo que aquellos necesitan. El enfoque paternalista, entre otros aspectos, ahorra el esfuerzo que supone salir del aula y escuchar a los otros. Sin embargo, un periodista, cargado con los criterios aprendidos de noticiabilidad y con los prejuicios sin cuestionar, no debería imponer su visión y dejar de dialogar con las comunidades de referencia atendiendo a su diversidad. Escuchar activamente es hacerse cargo de los discursos y perspectivas de los actores implicados. Los grupos de trabajo deben hacer frente a la incertidumbre de no saber qué se les está pidiendo con claridad, porque no hay una respuesta preestablecida, pero también cabe la sorpresa y la motivación que deriva de la posibilidad de aportar algo, de iniciar un proyecto con sentido.

\section{Proponemos un enfoque que recupere el protagonismo de la dimensión social del periodismo}

Uno de los ejemplos de éxito fue el proyecto sobre espacios de cultura en Cuenca. El grupo tuvo que desistir de su idea inicial (proyectos culturales para la recuperación de espacios abandonados), después de descubrir las múltiples trabas económicas y burocráticas que el proyecto debía hacer frente. Pero en el proceso de indagación y escucha de colectivos culturales se descubrió que grupos de jóvenes de la ciudad necesitaban espacios para ensayar y promocionar sus expresiones culturales (música, circo, baile). En este caso los periodistas no llevaron a cabo una campaña de concienciación, sino que sirvieron de hilo conductor, actuaron como mediadores y animaron a los colectivos a hacer una petición conjunta al ayuntamiento usando las herramientas comunicativas a su disposición. No sólo se grabó un videoclip para lanzar la petición y visibilizar una comunidad que tenía poca presencia en la ciudad, sino que los comunicadores fueron los mediadores para la conformación de una red.

El último paso de estos proyectos consiste en diseñar un prototipo que dé cuenta de la propuesta que se ha dado al reto o al problema; puede ser una solución o, de forma más modesta, una aportación en su avance. En esta fase se despliegan todas las herramientas y destrezas aprendidas durante los cursos anteriores de Grado, ahora sí, enfocadas en un producto comunicativo o resultado específico. 


\section{Conclusiones}

Poniendo el énfasis en la diferencia entre perfil y función (Saiz; Galletero, 2016), proponemos un enfoque que recupere el protagonismo de la dimensión social del periodismo, las competencias actitudinales y los valores éticos, con el fin de desarrollar la capacidad mediadora de los periodistas en las sociedades democráticas. La sinergia del periodismo con otras áreas como puede ser la de trabajo social abren un nuevo campo de experimentación, más allá de las innovaciones derivadas de lo tecnológico.

El aprendizaje por proyectos y la metodología de estudio de caso anima a los estudiantes a enfrentarse a un reto con el riesgo de no saber a ciencia cierta qué se conseguirá al final. Al situar el foco en el proceso y no en el resultado, se transita desde el autoanálisis, la tolerancia a la incertidumbre y la frustración, la perseverancia y la paciencia, hasta la escucha activa, la capacidad de empatía y la apertura y el respeto hacia los otros. Esta capacidad posibilitará que la atención a los colectivos vulnerables no quede restringida a una tarea asistencial, fundamentada en enfoques alternativos y minoritarios, sino que se adapte a un modelo con vocación innovadora y legitimada. Volviendo a Kapucinsky (2003, p. 16), este método de trabajo incide sobre la necesidad de recordar que

"la condición fundamental de este oficio es el entendimiento con el otro: hacemos y somos aquello que los otros nos permiten".

La experiencia real ha demostrado que los estudiantes reflexionan sobre otro modo de concebir los nuevos medios sociales, a veces guiados por el espectáculo y la banalidad. Al mismo tiempo ponen a prueba sus conocimientos en base a casos concretos, produciéndose así una simbiosis entre el mundo académico y su contexto más cercano. La posibilidad de poder modificar situaciones que les rodean o contribuir a la participación ciudadana de manera directa funciona como un elemento motivador muy eficaz.

La sinergia del periodismo con otras áreas como el trabajo social abren un nuevo campo de experimentación

\section{Bibliografía}

Aibar, Eduardo (1996). “La vida social de las máquinas: orígenes, desarrollo y perspectivas actuales en la sociología de la tecnología". Reis, v. 76, n. 96, pp. 141-170. http://www.reis.cis.es/REIS/PDF/REIS_076_09.pdf

Aibar, Eduardo (2001). “Fatalismo y tecnología. ¿Es autónomo el desarrollo tecnológico?". UOC, julio. http://www.uoc.edu/web/esp/art/uoc/0107026/aibar.htm/

Aguilar-Idáñez, María-José (2016). “Comunicación social: la gran ausente en la formación social". María-José AguilarIdañez, 2 abril.

https://mariajoseaguilaridanez.wordpress.com/2016/04/02/ comunicacion-social-la-gran-ausente-en-la-formacion-social
Aneca (2004). Libro blanco. Título de Grado en Trabajo Social. Madrid: Aneca.

http://www.aneca.es/var/media/150376/libroblanco_ trbjsocial_def.pdf

Aneca (2005). Libro blanco. Títulos de Grado en Comunicación. Madrid: Aneca.

http://www.aneca.es/var/media/150336/libroblanco_ comunicacion_def.pdf

Arriazu-Muñoz, Rubén (2015). "La incidencia de la brecha digital y la exclusión social tecnológica. El impacto de las competencias digitales en los colectivos vulnerables". Praxis sociológica, n. 19, pp. 225-240.

http://www.praxissociologica.es/images/PDF/2015-19/ praxis19-13.pdf

Bassets, Lluís (2013). El último que apague la luz: sobre la extinción del periodismo. Madrid: Taurus. ISBN: 978 8430602094

Bolter, Jay-David; Grusin, Richard (2000). Remediation. Understanding new media. Cambridge: MIT Press. ISBN: 0262522799 https://goo.gl/udde1g

Casero-Ripollés, Andreu; Ortells-Badenes, Sara; Doménech-Fabregat, Hugo (2013). "Las competencias profesionales en periodismo: una evaluación comparativa". Historia y comunicación social, v. 18, pp. 53-64. https://doi.org/10.5209/rev_HICS.2013.v18.44311

Castelló-Martínez, Araceli (2010). “Una nueva figura profesional: el community manager". Revista Pangea, v. 1, pp. 74-97.

https://revistaraic.wordpress.com/2010/09/15/01-01-104

Castells, Manuel (2009). Comunicación y poder. Madrid: Alianza Editorial. ISBN: 9788420684994

Castells, Manuel (2013). El impacto de internet en la sociedad: una perspectiva global. España: BBVA. https://goo.gl/V8hylf

Espiritusanto, Óscar; Gonzalo-Rodríguez, Paula (2011). Periodismo ciudadano. Evolución positiva de la comunicación. Madrid: Fundación Telefónica, Ariel. ISBN: 9788408104162 http://www.fundaciontelefonica.com/arte_cultura/ publicaciones-listado/pagina-item-publicaciones/itempubli/125

Fuente-Cobo, Carmen; Mera-Fernández, Montse (2013). “Implantación del método del caso en la enseñanza del periodismo en las universidades españolas: un análisis a partir de las guías docentes". Estudios sobre el mensaje periodístico, v. 19 , pp. 177-186.

https://doi.org/10.5209/rev_ESMP.2013.v19.42023

Gámez-Fuentes, María-José; Nos-Aldás, Eloísa (2012). “Comunicación para la igualdad en el nuevo EEES: fundamentación crítica para el cambio social". Estudios sobre el mensaje periodístico, v. 18, pp. 325335.

https://doi.org/10.5209/rev_ESMP.2012.v18.40986

Gertrudis-Casado, María-Carmen; Gértrudix, Manuel; Álvarez-García, Sergio (2016). “Competencias informativas profesionales y datos abiertos. Retos para el empodera- 
miento ciudadano y el cambio social". Comunicar, v. $24, \mathrm{n}$. 47, pp. 39-47.

http://dx.doi.org/10.3916/C47-2016-04

Jarvis, Jeff (2015). El fin de los medios de comunicación de masas. ¿Cómo serán las noticias del futuro? Barcelona: Gestión 2000, Grupo Planeta. ISBN: 9788498754018

Kapucinski, Ryszard (2003). Los cinco sentidos del periodista. Madrid: Fondo de Cultura Económica. ISBN: 9681670914 https://reportepolitico.files.wordpress.com/2011/03/ kapuscinski_los_cinco_sentidos_del_periodista.pdf

Lafuente, Antonio; Corsín, Alberto (2015). “Economías y tecnologías del don. En: El procomún y los bienes comunes. Dossieres Economistas sin fronteras, n. 16.

http://ecosfron.org/ecosfron/wp-content/uploads/DOSSIERESEsF-16-El-procom\%C3\%BAn-y-los-bienes-comunes.pdf

Lafuente, Antonio; Lara, Tíscar (2013). "Aprendizajes situados y prácticas procomunales". Revista de la asociación de sociología de la educación, v. 6, n. 2, pp. 168-177. https://ojs.uv.es/index.php/RASE/article/view/8352/7945

Latour, Bruno (2008). Reensamblar lo social: una introducción a la teoría del actor-red. Buenos Aires: Manantial. ISBN: 9789875001145

https://seminariosocioantropologia.files.wordpress. com/2016/08/orca_share_media1470719009830-1.pdf

López-García, Guillermo (2015). Periodismo digital. Redes, audiencias y modelos de negocio. Salamanca: Comunicación Social, 170 pp. ISBN: 9788415544937

Mancini, Pablo (2012). Hackear el periodismo. Buenos Aires: La Crujía. ISBN: 9789876011341

Manfredi-Sánchez, Juan Luis (coord.) (2015). Innovación y periodismo: emprender en la universidad. Cuadernos artesanos de comunicación, n. 76. La Laguna (Tenerife): Sociedad Latina de Comunicación Social. ISBN: 9788415698937 http://dx.doi.org/10.4185/cac76

Martínez-Rodrigo, Estrella; Sánchez-Martín, Lourdes (2012). "Las competencias europeas del profesional de la comunicación en el Espacio Europeo de Educación Superior". En: Túñez-López, Miguel (coord.). Viejo periodismo, nuevos periodistas. Cuadernos Artesanos de Comunicación, n. 25. La Laguna (Tenerife): Sociedad Latina de Comunicación Social, pp. 125-136. ISBN: 9788494011108 http://www.revistalatinacs.org/067/cuadernos/25_ Viejo\%20periodismo.pdf

Nos-Aldás, Eloísa (2011). “Educación en medios hacia cul- turas de paz. Los futuros profesionales de la comunicación como ciudadanos mediadores". Enseñanza \& teaching, v. 29, n. 2, pp. 87-102.

http://campus.usal.es/ revistas_trabajo/index.php/02125374/article/viewFile/9255/9530

Nos-Aldás, Eloísa; Smith, Matt B. (coords.) (2016). Número monográfico: "Comunicación, sociedad civil y cambio social". Comunicar, v. 24, n. 47.

https://goo.gl/YhtY4V

Orozco-Gómez, Guillermo; Navarro-Martínez, Eva; GarcíaMatilla, Agustín (2012). "Desafíos educativos en tiempos de auto-comunicación masiva: la interlocución de las audiencias". Comunicar, v. 19, n. 38, pp. 67-74.

https://doi.org/10.3916/C38-2012-02-07

Riesco-González, Manuel (2008). “El enfoque por competencias en el EEES y sus implicaciones en la enseñanza y el aprendizaje". Tendencias pedagógicas, v. 13, pp. 79-115.

http://hdl.handle.net/10486/4704

Saiz, Vanesa; Galletero, Belén (2016). “Los periodistas de Castilla-La Mancha: una aproximación al entorno laboral postcrisis". En: I Congreso iberoamericano Nebrija Piatcom. Madrid.

Salaverría, Ramón (2015). "Los labs como fórmula de innovación en los medios". El profesional de la información, v. 24, n. 4, pp. 397-404.

http://dx.doi.org/10.3145/epi.2015.jul.06

Sánchez-García, Pilar; Campos-Domínguez, Eva; BerrocalGonzalo, Salomé (2015). "Las funciones inalterables del periodista ante los perfiles multimedia emergentes". Revista latina de comunicación social, n. 70, pp. 187-208.

http://dx.doi.org/10.4185/RLCS-2015-1042

Scolari; Carlos-Alberto; Micó-Sanz, Josep-Lluís; NavarroGuere, Héctor; Pardo-Kuklinski, Hugo (2008). “El periodista polivalente. Transformaciones en el perfil del periodista a partir de la digitalización de los medios audiovisuales catalanes". ZER, v. 13, n. 25, pp. 37-60.

http://www.ehu.eus/zer/hemeroteca/pdfs/zer25-02scolari.pdf

Tuñez-López, Miguel; Martínez-Solana, Yolanda; AbejónMendoza, Paloma (2010). "Nuevos entornos, nuevas demandas, nuevos periodistas". Estudios sobre el mensaje periodístico, v. 16, p. 79-94.

http://revistas.ucm.es/index.php/ESMP/article/view/ ESMP1010110079A/11358
El profesional de la -información-

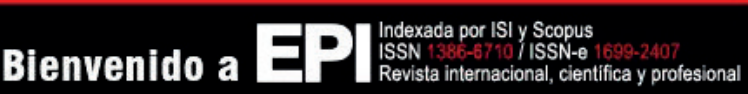

http://www.elprofesionaldelainformacion.com

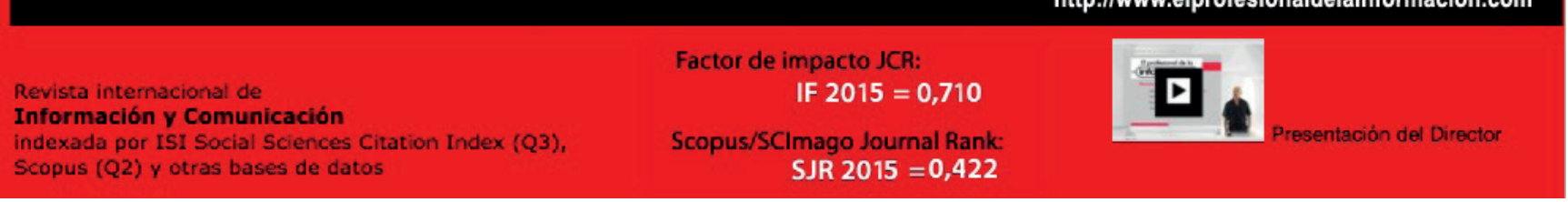

